\title{
Effects of miR-145 on the inhibition of chondrocyte proliferation and fibrosis by targeting TNFRSF11B in human osteoarthritis
}

\author{
GUO-DONG WANG ${ }^{1,2}$, XIAO-WEI ZHAO ${ }^{2}$, YU-GE ZHANG ${ }^{2}$, YING KONG ${ }^{2}$, \\ SHUAI-SHUAI NIU ${ }^{2}$, LONG-FEI MA ${ }^{2}$ and YUAN-MIN ZHANG ${ }^{2}$ \\ ${ }^{1}$ Qingdao University, Qingdao, Shandong 266000; ${ }^{2}$ Department of Orthopaedics, \\ The Affiliated Hospital of Jining Medical University, Jining, Shandong 272029, P.R. China
}

Received September 19, 2015; Accepted October 3, 2016

DOI: $10.3892 / \mathrm{mmr} .2016 .5981$

\begin{abstract}
Osteoarthritis (OA) is a common cause of functional deterioration in older adults, and altered chondrogenesis is the most common pathophysiological process involved in the development of OA. MicroRNA-145 (miR-145) has been shown to regulate chondrocyte homeostasis. However, the function of miR-145 in OA remains to be elucidated. In the present study, the expression levels of miR-145 were examined in cartilage specimens from 25 patients with knee OA using reverse transcription-quantitative polymerase chain reaction analysis. The effects of miR-145 on the proliferation and fibrosis of the C-20/A4 and CH8 cell lines were also investigated using 3-(4,5-dimethylth-iazol-2-yl)-2,5-diphenyltetrazolium bromide and western blot assays in vitro. The results revealed that the expression of miR-145 was decreased in the OA cartilage tissues, compared with normal cartilage tissues. The overexpression of miR-145 by transfection of cells with miR-145 mimics significantly inhibited C-20/A4 and CH8 cell proliferation and fibrosis. Furthermore, tumor necrosis factor receptor superfamily, member 11b (TNFRSF11B) was identified as a direct target of miR-145 in chondrocytes, which was confirmed using a dual-luciferase reporter assay. The expression level of TNFRSF11B was markedly upregulated in the patients with OA, and the ectopic expression of miR-145 was capable of suppressing the expression of TNFRSF11B. In addition, the knock down of TNFRSF11B using specific small interfering RNA also inhibited the proliferation and fibrosis of C-20/A4 and CH8 cells in vitro. These data provide the first evidence, to the best of our knowledge, to suggest the critical function of miR-145 in regulating the expression of TNFRSF11B, which may have important implications on the regulation of chondrocyte proliferation and fibrosis in OA.
\end{abstract}

Correspondence to: Professor Yuan-Min Zhang, Department of Orthopaedics, The Affiliated Hospital of Jining Medical University, 89 Guhuai Road, Jining, Shandong 272029, P.R. China

E-mail: ymzyeardr@sina.com

Key words: osteoarthritis, microRNA-145, proliferation, fibrosis, tumor necrosis factor receptor superfamily, member $11 \mathrm{~b}$

\section{Introduction}

Osteoarthritis (OA) is the most common form of musculoskeletal disease (1). It is a complex and multifaceted disease, which is predominantly characterized by the degradation of articular cartilage and joint inflammation (2). Chondrocytes in the articular cartilage regulate appropriate gene expression in order to achieve tissue homeostasis. At present, although a number of pathways underlying the pathogenesis of OA have been demonstrated, it remains to be fully elucidated and current knowledge has not provided effective approaches for prevention or treatment.

MicroRNAs (miRNAs) are small non-coding RNAs, which have been reported to be important regulators of gene expression in humans and function as negative regulator of gene expression at the post-transcriptional level via binding to complimentary sequences in the 3'-untranslated regions (3'-UTRs) of targeting mRNAs $(3,4)$. Hundreds of miRNAs have been found in various organisms, and a third of all mammalian mRNAs appear to be under the regulation of miRNAs (5). Aberrant miRNA expression profiles have been demonstrated to be associated with the development of OA, and their functions are beginning to be delineated (6-8).

miR-145 has been indicated to be important in tumor progression and metastasis, specifically in the processes of cell proliferation and differentiation (9-11). However, the role of miR-145 in the progression of OA and its underlying mechanism remains to be fully elucidated. The present study aimed to demonstrate the biological function and molecular mechanism of miR-145 in OA. The expression of miR-145 was found to decrease and that of tumor necrosis factor receptor superfamily, member 11b (TNFRSF11B) was found to increase in OA cartilage tissues, compared with normal cartilage tissues. It was also revealed that miR-145 was important in chondrocyte proliferation and fibrosis by directly targeting TNFRSF11B, which inhibited the proliferation and fibrosis of $\mathrm{OA}$.

\section{Materials and methods}

Cell lines and patient samples. The C-20/A4 and CH8 human articular chondrocytes cell lines were obtained from American Type Culture Collection (Manassas, VA, USA). All cells were 
cultured in Dulbecco's modified Eagle's medium supplemented with $10 \%$ fetal bovine serum in a humidified atmosphere of $5 \% \mathrm{CO}_{2}$ at $37^{\circ} \mathrm{C}$. A total of 25 paired cartilage tissues, and matched normal cartilage tissues from traumatic amputees were obtained from the knees of patients with OA. Each patient provided consent for the experiment, and the present study was approved by the Ethics Committee of the Affiliated Hospital of Jining Medical University (Jining, China).

RNA extraction and reverse transcription-quantitative polymerase chain reaction $(R T-q P C R)$ analysis. Total RNA from the tissues and cells were extracted using TRIzol reagent (Invitrogen; Thermo Fisher Scientific, Inc., Waltham, MA, USA) and purified using the RNeasy Maxi kit (Qiagen GmbH, Hilden, Germany) according to the manufacturer's protocol. The miScript reverse transcription kit (Qiagen $\mathrm{GmbH}$ ) and RevertAid First Strand cDNA Synthesis kit (Qiagen GmbH) were used to obtain the total cDNA. The miScript SYBR Green PCR kit (Qiagen GmbH) and SYBR ${ }^{\circledR}$ Premix Ex Taq ${ }^{\mathrm{TM}}$ II (Takara Bio, Inc., Otsu, Japan) were used for the qPCR assay. The PCR reaction mixture contained: $1 \mu \mathrm{l}$ cDNA, $2 \mu \mathrm{l}$ specific primer, $2 \mu 1$ 10X miScript SYBR Green PCR Master Mix (or SYBR $^{\circledR}$ Premix Ex Taq ${ }^{\mathrm{TM}}$ II) and $5 \mu 1$ RNase-free water. The amplification conditions were as follows: $95^{\circ} \mathrm{C}$ for $10 \mathrm{~min}$, followed by 45 cycles at $95^{\circ} \mathrm{C}$ for $30 \mathrm{sec}, 60^{\circ} \mathrm{C}$ for $30 \mathrm{sec}$ and $72^{\circ} \mathrm{C}$ for $30 \mathrm{sec}$, and a final extension step at $72^{\circ} \mathrm{C}$ for $5 \mathrm{~min}$. Human U6 and GAPDH were used as the internal control genes. The miR-145- and U6-specific primers were as follows: miR-145 forward 5'-GAATCCCTTAGATGCTAAGATG-3' and reverse (miScript SYBR Green PCR kit universal primer); U6, forward 5'-CTCGCTTCGGCAGCACA-3' and reverse 5'-ACGCTTCACGAATTTGCGT-3'. The TNFRSF11B- and GAPDH-specific primers were as follows: TNFRSF11B, forward 5'-CCCCTTGCCCTGACCACTACTA-3' and reverse 5'-CGATTGCACTCCTGCTTGACGT-3'; GAPDH, forward 5'-ACATCAAGAAGGTGGTGAAGCAGG-3' and reverse 5'-AGCGTCAAAGGTGGAGGAGTGG-3'. The relative expression level was determined using the delta-delta $\mathrm{Cq}$ method (12).

Cell transfection. The miR-145 mimics, negative control (NC) mimics, small interfering (si)TNFRSF11B and negative control were designed and synthesized by Invitrogen Life Technologies; Thermo Fisher Scientific, Inc. The sequence of the miR-145 mimic was 5'-GUCCAGUUUUCCCAGGAA UCCCU-3' and that of the NC mimic was 5'-UUCUCCGAA CGUGUCACGUUU-3'. The sequence of siTNFRSF11B was 5'-CAGGCACUUGAGGCUUUCAGUGAUA-3' and that of the NC was 5'-CAGUACUUUUGUGUAGUACAA-3'. Cell transfection was performed using Lipofectamine 2000 (Invitrogen; Thermo Fisher Scientific, Inc.), according to the manufacturer's protocols.

In vitro cell proliferation assay. Cell proliferation was measured using a 3-(4,5-dimethylth-iazol-2-yl)-2,5-diphenyltetrazolium bromide (MTT) assay. The C-20/A4 and CH8 cells $\left(\sim 4 \times 10^{3}\right)$ were seeded into a 96 -well plate and transfected with the miR-145 or NC mimics, and siTNFRSF11B or the $\mathrm{NC}$ at $37^{\circ} \mathrm{C}$. At $0,24,48$ or 72 h-post transfection, $25 \mu \mathrm{l}$ of MTT reagent $(5 \mathrm{mg} / \mathrm{ml}$; Sigma-Aldrich; Merck Millipore,
Darmstadt, Germany) was added to each plate and incubated at $37^{\circ} \mathrm{C}$ for $4 \mathrm{~h}$. Subsequently, the MTT medium mixture was discarded and $150 \mu \mathrm{l}$ of dimethyl sulfoxide was added to each plate and agitated for $30 \mathrm{~min}$ at $37^{\circ} \mathrm{C}$ to solubilize the crystals. Absorbance was measured at a wavelength of $450 \mathrm{~nm}$ using an ELISA microplate reader (Bio-Rad Laboratories, Inc., CA, USA).

Dual-luciferase reporter assay. In order to confirm whether miR-145 was able to directly bind the 3'-UTR of the TNFRSF11B gene, the miR-145 target sequences of TNFRSF11B were inserted between the Xhol and NotI restriction sites of the 3'-UTR of the hRluc gene in the psiCHeCK ${ }^{\mathrm{TM}}-2$ luciferase vector (Promega Corporation, Madison, WI, USA). Primer sequences for the 3'-UTR of TNFRSF11B mRNA were designed by Thermo Fisher Scientific, Inc., as follows: Forward 5'-CTCGCTTCGGCAGCACA-3' and reverse 5'-ACGCTT CACGAATTTGCGT-3'. The recombination plasmid of psiCHECK-2-TNFRSF11B-3'-UTR was constructed, which contained the potential binding sites, and the potential binding sites were manually mutated by exchanging the $\mathrm{G}$ and $\mathrm{T}, \mathrm{A}$ and C. Subsequently, the luciferase recombination reporter constructs, together with the miR-145 mimics or NC mimics, were cotransfected into the C-20/A4 and $\mathrm{CH} 8$ cells at $37^{\circ} \mathrm{C}$. The Firefly and Renilla luciferase activities were detected using a dual luciferase assay system (Promega Corporation), according to the manufacturer's protocol, $24 \mathrm{~h}$ following transfection. Normalized data were calculated as the quotient of Renilla/Firefly luciferase activities.

Western blot analysis. Total proteins of the chondrocyte tissues and cells were extracted using RIPA buffer (Invitrogen; Thermo Fisher Scientific, Inc.) supplemented with protease inhibitor cocktail (EMD Millipore, Billerica, MA, USA). Protein concentration was determined using the Bio-Rad Protein Assay Dye Reagent Concentrate (Bio-Rad Laboratories, Inc., Hercules, CA, USA). The extracted proteins $(30 \mu \mathrm{g})$ were separated by $12 \%$ SDS-PAGE and transferred onto a polyvinylidene difluoride membrane (EMD Millipore). The membranes were blocked with $5 \%$ non-fat dried milk for $2 \mathrm{~h}$ at $37^{\circ} \mathrm{C}$. Subsequently, the membranes were incubated with mouse anti-TNFRSF11B monoclonal antibody (cat. no. ab105935), mouse anti-collagen II monoclonal antibody (cat. no. ab3092), mouse anti-collagens V monoclonal antibody (cat. no. ab112551), mouse anti-collagen X monoclonal antibody (cat. no. ab49945), mouse anti-GAPDH monoclonal antibody (cat. no. ab8245), rabbit anti-matrix metalloproteinase (MMP)1 monoclonal antibody (cat. no. ab52631), rabbit anti-MMP8 monoclonal antibody (cat. no. ab81286) and rabbit anti-MMP13 monoclonal antibody (cat. no. ab51072), all from Abcam (Cambridge, MA, USA), overnight at $4^{\circ} \mathrm{C}$. Following incubation, the membranes were treated with horseradish peroxidase-labeled secondary antibodies (cat. nos. ab6728 and ab6721; Abcam) for $1 \mathrm{~h}$ at room temperature. The positive blots were detected using a chemiluminescent substrate kit (Pierce; Thermo Fisher Scientific, Inc.).

Statistical analysis. Statistical analysis was performed using SPSS 17.0 (SPSS, Inc., Chicago, IL, USA). Data are presented as the mean \pm standard deviation. Statistical significance was 


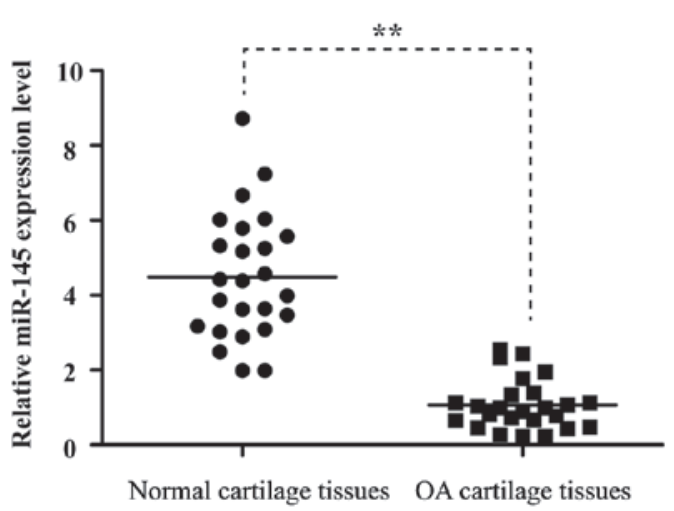

Figure 1. Expression of miR-145 is decreased in OA cartilage tissues, compared with normal cartilage tissues. Reverse transcription-quantitative polymerase chain reaction analysis was performed to examine the expression levels of miR-145 in OA cartilage and normal cartilage tissues. Data are expressed as the mean \pm standard deviation $(n=3)$. ${ }^{* *} \mathrm{P}<0.01$. miR, microRNA; OA, osteoarthritis.

determined using Student's $t$-test, with the exception of the MTT data, which was analyzed using one-way analysis of variance followed by Bonferroni multiple comparisons test. $\mathrm{P}<0.05$ was considered to indicate a statistically significant difference. All experiments were repeated at least three times.

\section{Results}

Expression of miR-145 is decreased in OA cartilage tissues, compared with normal cartilage tissues. To determine whether the expression of miR-145 is altered in OA cartilage tissues, the present study compared its level of expression between knee OA and normal cartilage tissues. As shown in Fig. 1, the expression level of miR-145 was significantly reduced in the OA cartilage tissues, compared with the normal cartilage tissues.

Overexpression of miR-145 induces the arrest of chondrocyte proliferation and inhibits chondrocyte fibrosis. To investigate the function of miR-145 in cell proliferation, miR-145 mimics or NC mimics were transfected into $\mathrm{C}-20 / \mathrm{A} 4$ and $\mathrm{CH} 8$ cells. The results of the MTT assay demonstrated that the cell proliferation in the miR-145 mimic group was significantly decreased, compared with that in the NC mimic group (Fig. 2A). In order to examine whether miR-145 also regulated chondrocyte fibrosis, western blot analysis was performed to examine the human C-20/A4 and CH8 cells transfected with miR-145 or $\mathrm{NC}$ mimics. It was found that the protein expression levels of collagen II, $\mathrm{V}$ and $\mathrm{X}$ in the miR-145 mimic group were upregulated, whereas the protein expression levels of MMP1, MMP8 and MMP13 were downregulated (Fig. 2B), compared with the levels in the NC mimic group. These results suggested that miR-145 inhibited chondrocyte proliferation and fibrosis.

TNFRSF11B is a direct target of miR-145. miRanda (http://www. microrna.org) and miRcode (http://www.mircode.org) were used to predict the downstream target genes of miR-145. TNFRSF11B was one of the putative genes identified, the mRNA 3'-UTR of which contained a complementary site for the seed region of miR-145 (Fig. 3A). In order to confirm the
A
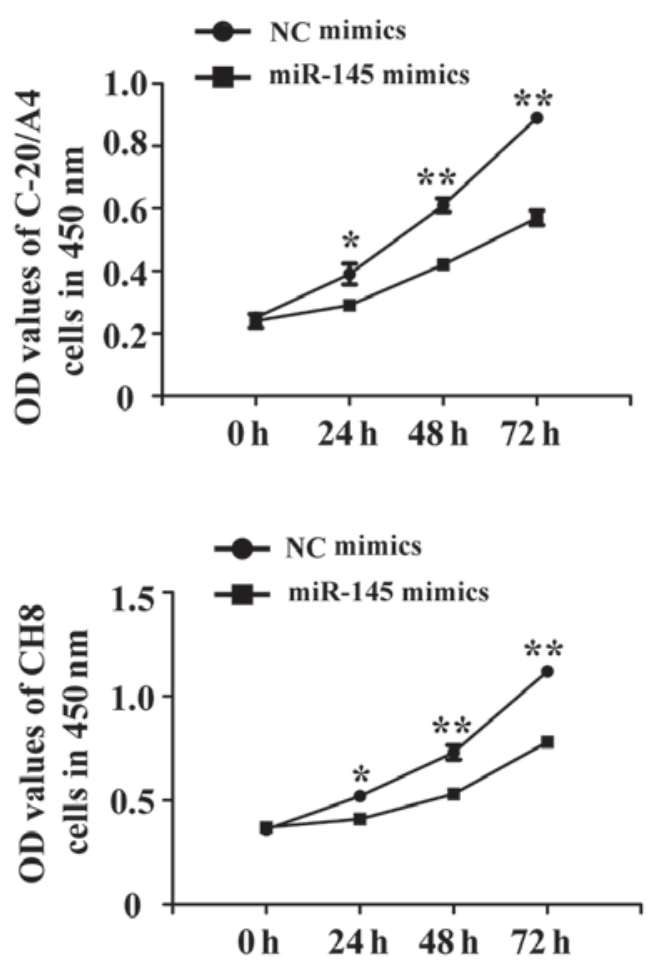

$\mathbf{B}$
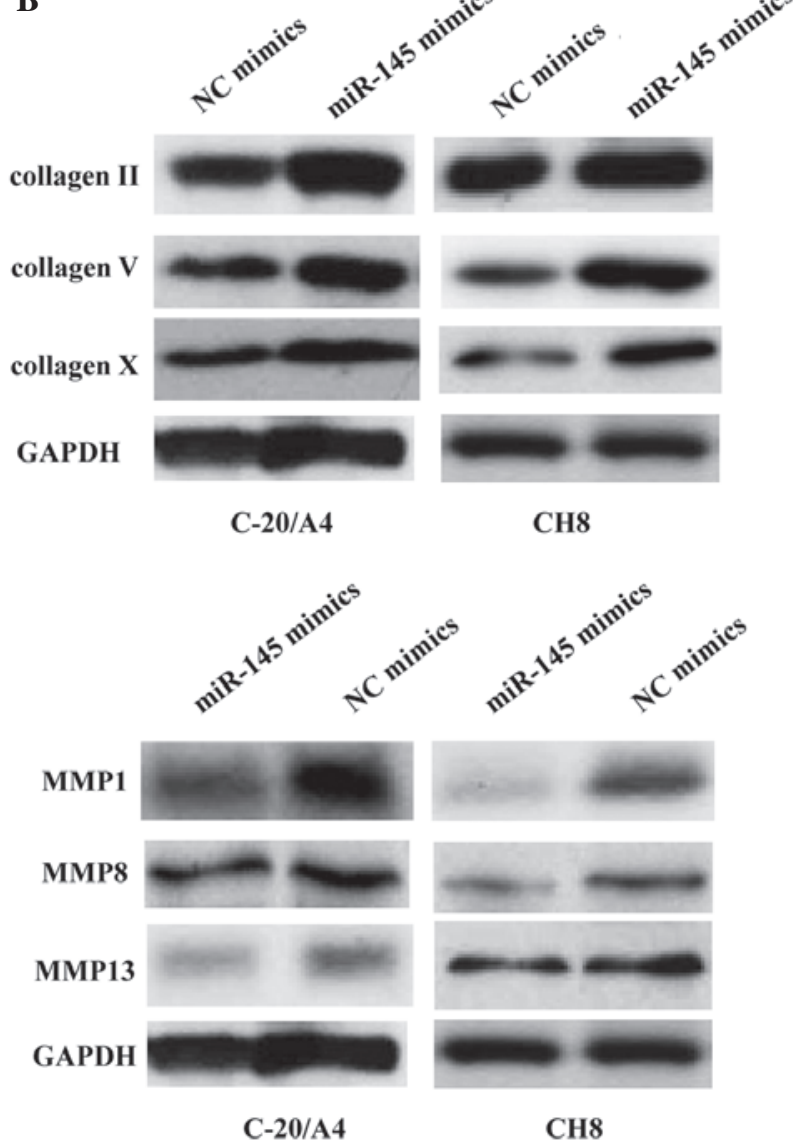

Figure 2. Overexpression of miR-145 induces proliferation arrest and inhibits fibrosis of chondrocytes. (A) At 24, 48, 36 and $72 \mathrm{~h}$ post-transfection, a 3-(4,5-dimethylth-iazol-2-yl)-2,5-diphenyltetrazolium bromide assay was performed to detect cell proliferation. ${ }^{*} \mathrm{P}<0.05$ and ${ }^{* *} \mathrm{P}<0.01$. (B) Western blot analysis was performed to detect the protein expression of collagen II, V and X, and MMP1, MMP8 and MMP13 in C-20/A4 and CH8 cells following transfection with miR-145 or NC mimics. miR, microRNA; MMP, matrix metalloproteinase; OD, optical density; NC, negative control. 
A

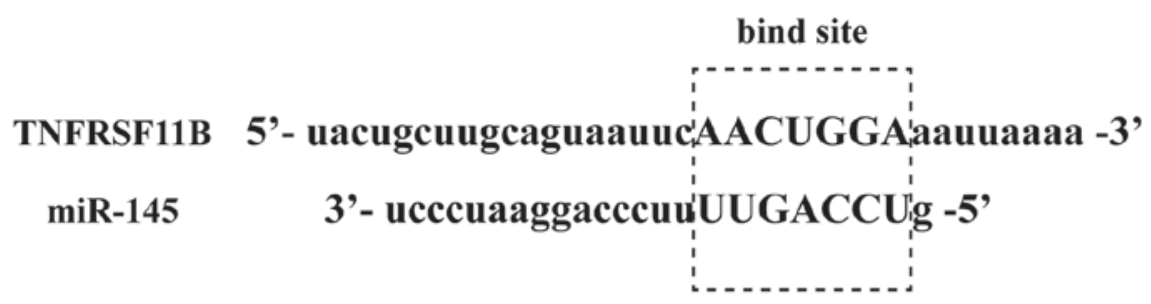

B

TNFRSF11B (WT) 5'- uacugcuugcaguaauucAACUGGAaauuaaaa -3'

TNFRSF11B (MUT) 5'- uacugcuugcaguaauucCCAGTTCaauuaaaa -3'
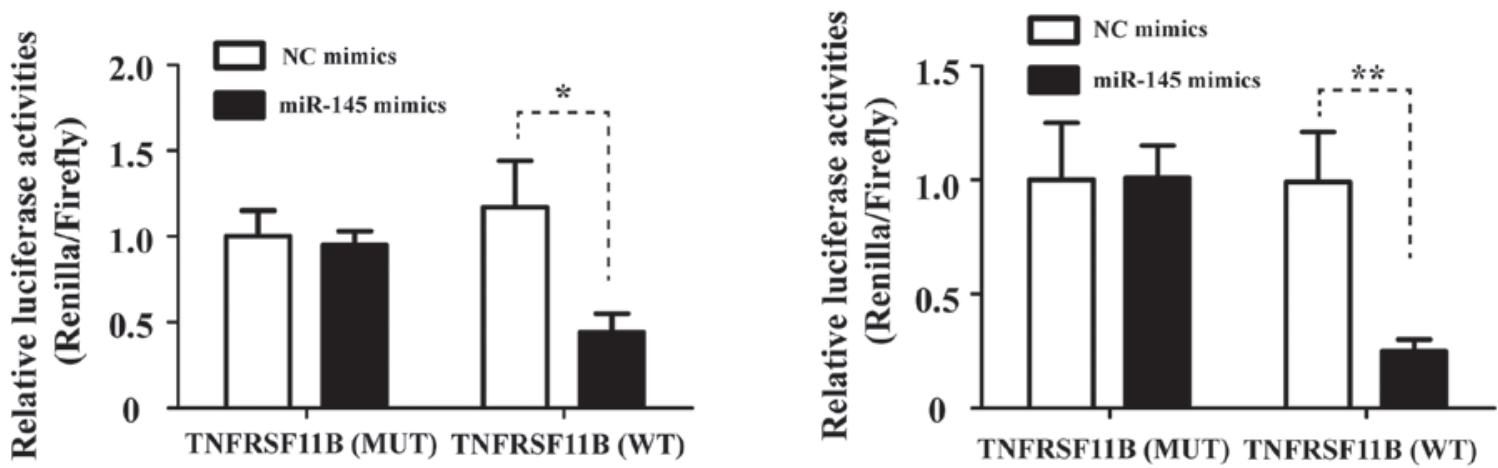

C
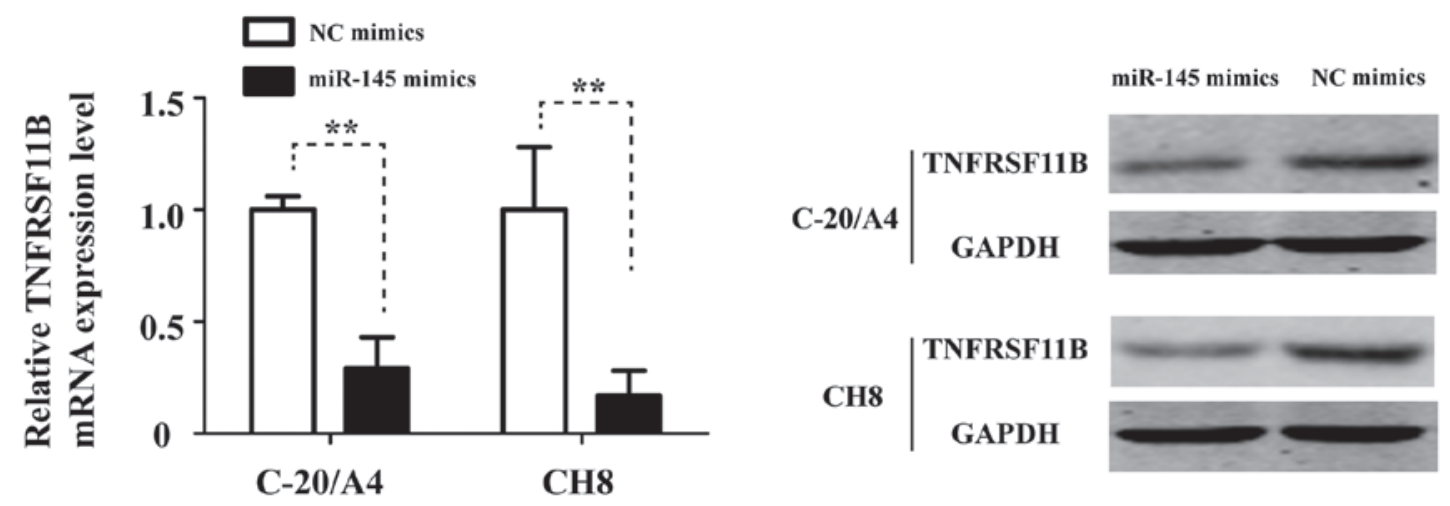

Figure 3. TNFRSF11B is a direct target of miR-145. (A) TNFRSF11B mRNA 3'-UTRs showing the target binding sites for miR-145. (B) C-20/A4 and CH8 cells were cotransfected with miR-145 mimics or NC mimics and a TNFRSF11B-3'-untranslated region fragment containing either the WT miR-145 target sequence or MUT sequence. (C) mRNA and protein expression of TNFRSF11B, detected using reverse transcription-quantitative polymerase chain reaction and western blot analyses, respectively, in C-20/A4 and CH8 cells transfected with miR-145 mimics or NC mimics. GAPDH was used as an internal control. All experiments were performed three times, and a representative results are shown. ${ }^{*} \mathrm{P}<0.05$ and ${ }^{* * *} \mathrm{P}<0.01$. miR, microRNA; TNFRSF11B, tumor necrosis factor receptor superfamily, member 11b; WT, wild-type; MUT, mutant; NC, negative control.

direct targeting of TNFRSF11B by miR-145, wild-type (WT) TNFRSF11B-3'-UTR, containing the target sequences, or mutant (MUT) TNFRSF11B-3'-UTR mimics were constructed and inserted into a luciferase reporter vector to detect the effects of miR-145 on luciferase activity in C-20/A4 and CH8 cells. As shown in Fig. 3B, miR-145 suppressed the luciferase activity of the WT-TNFRSF11B-3'-UTR, whereas mutation of the miR-145 binding sites inhibited this suppressive effect. Furthermore, RT-qPCR and western blot analyses demonstrated that transfection with miR-145 mimics inhibited the endogenous expression of TNFRSF11B in the C-20/A4 and CH8 cells (Fig. 3C). These data suggested that miR-145 regulated the expression of TNFRSF11B by directly targeting its mRNA 3'-UTR in chondrocytes.
The knock down of TNFRSF11B also inhibited chondrocyte proliferation and fibrosis. To confirm the functional role of TNFRSF11B in chondrocytes, TNFRSF11B was knocked down in C-20/A4 and CH8 cells using TNFRSF11B-specific siRNAs. RT-qPCR and western blot analyses were then used to confirm the effect of this interference (Fig. 4A). The knockdown of TNFRSF11B significantly suppressed cell proliferation in the C-20/A4 and CH8 cells (Fig. 4B), compared with the $\mathrm{NC}$ group. The protein expression levels of collagen II, V and $\mathrm{X}$ were upregulated in the siTNFRSF11B group (Fig. 4C), whereas the protein expression levels of MMP1, MMP8 and MMP13 were downregulated (Fig. 4C) compared with the in the NC group. Taken together, these results demonstrated that miR-145 regulated the proliferation 
A
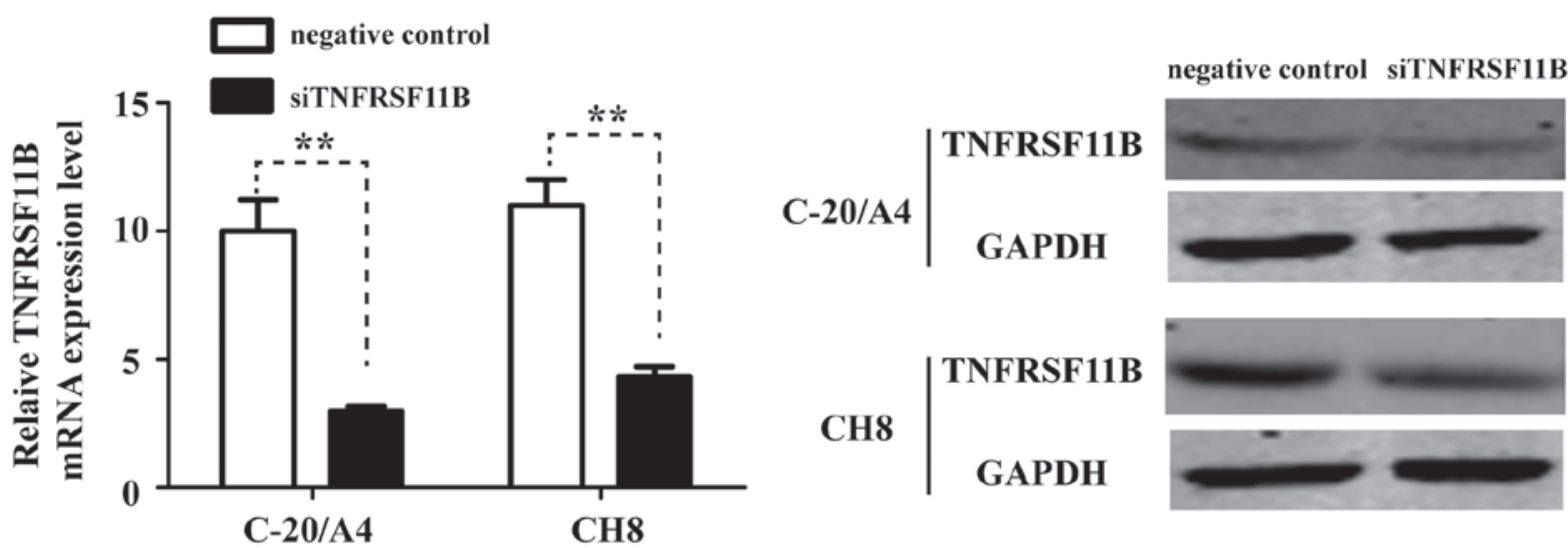

B
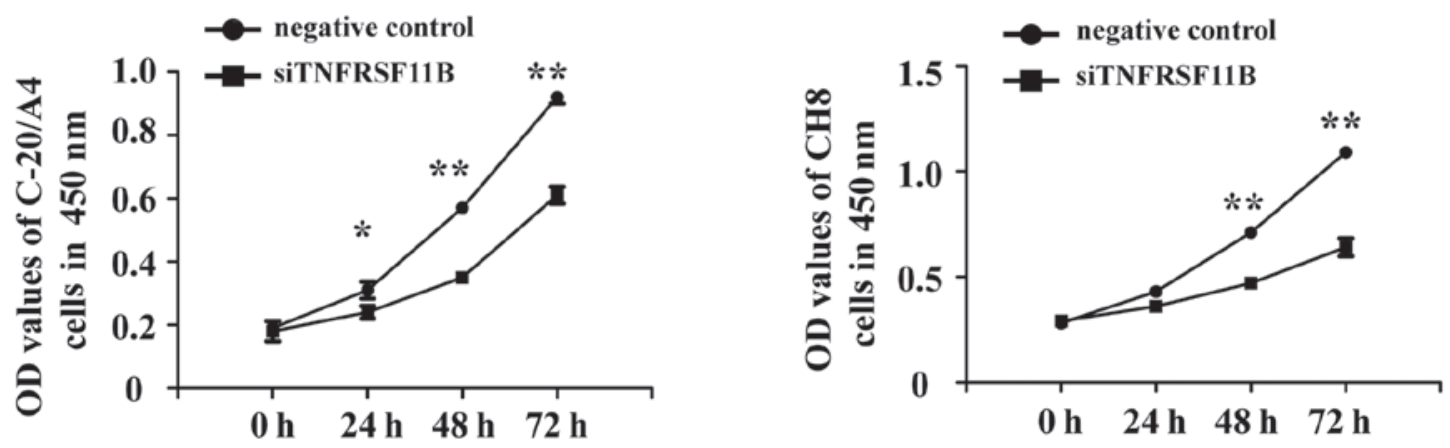

C
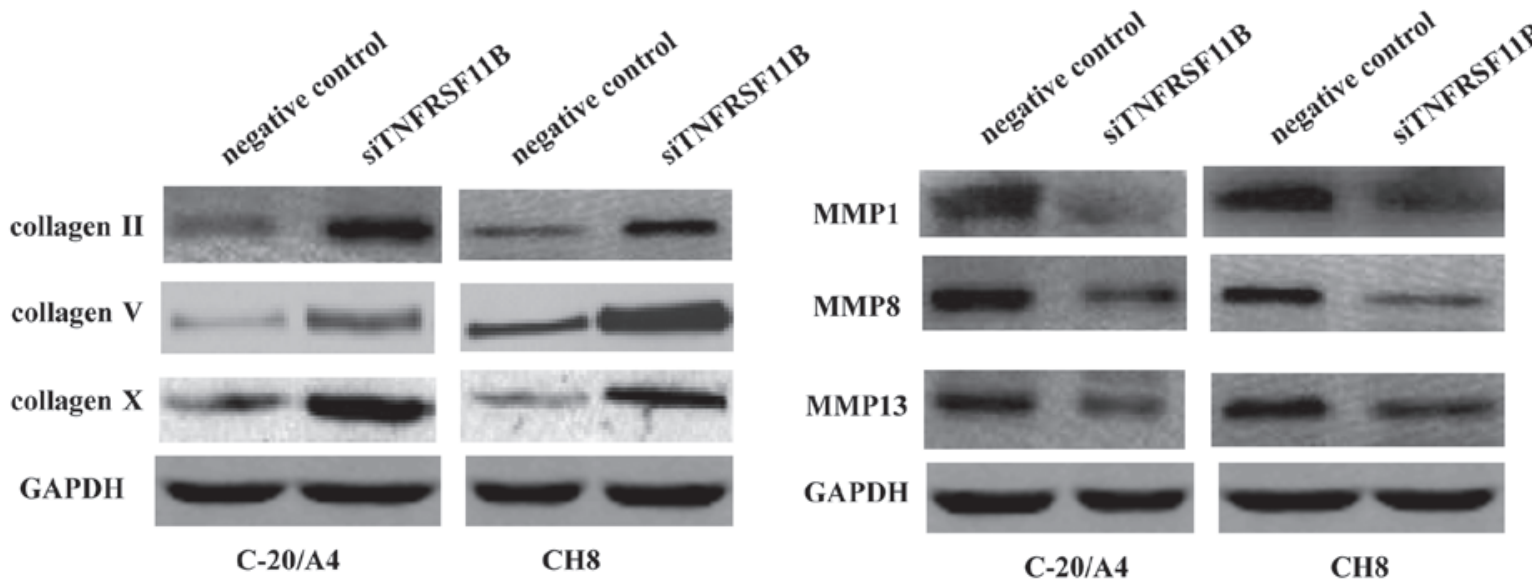

Figure 4. Knock down of TNFRSF11B inhibits chondrocyte proliferation and fibrosis. (A) Revesrse tranascription-quantitative polymerase chain reaction and western blot analyses were performed to examine the expression levels of TNFRSF11B in C-20/A4 and CH8 cells transfected with siTNFRSF11B or negative control. (B) A 3-(4,5-dimethylth-iazol-2-yl)-2,5-diphenyltetrazolium bromide assay was performed to examine the proliferation of C-20/A4 and CH8 cells transfected with siTNFRSF11B or negative control. ${ }^{*} \mathrm{P}<0.05$ and ${ }^{* *} \mathrm{P}<0.01$. (C) Western blot analysis was used to detect protein expression of collagen II, V and X, and MMP1, MMP8 and MMP13 in C-20/A4 and CH8 cells transfected with siTNFRSF11B or the negative control. siTNFRSF11B, small interfering RNA for tumor necrosis factor receptor superfamily, member 11b; NC, negative control; MMP, matrix metalloproteinase; OD, optical density.

and fibrosis of chondrocytes by targeting TNFRSF11B in human OA.

\section{Discussion}

miRNAs are essential in maintaining and modulating normal physiological function, however, the expression of miRNAs are altered in response to pathological disorders (13). OA is a prevalent degenerative joint disease, which is characterized by the progressive destruction of articular cartilage, synovial hyperplasia and the sclerosis of subchondral bone (14-17).
Previous studies have demonstrated that miRNAs are have a vital function in OA (6-8). Thus, it is important to detect the aberrant expression of miRNAs in OA. The present study indicated that miR-145 is essential in the development of OA. The expression of miR-145 was significantly decreased in human OA tissues. Transfection with a synthetic miR-145 mimic in vitro led to significant inhibition of the proliferation and fibrosis of C-20/A4 and CH8 cells.

In addition, bioinformatics analysis was used to screen the targets of miR-145, and suggested that TNFRSF11B may be a target of miR-145 in chondrocytes. The results of 
the dual-luciferase reporter assay further suggested that TNFRSF11B was a direct target of miR-145 in chondrocytes. TNFRSF11B, as a key regulator of the process of chondrogenesis, has been reported to be important in chondrocyte formation (18-20). In the present study, the overexpression of miR-145 repressed the expression of TNFRSF11B in C-20/A4 and $\mathrm{CH} 8$ cells and, as expected, interference of the expression of TNFRSF11B decreased chondrocyte growth and fibrosis.

To the best of our knowledge, the present study is the first to provide evidence of the function of miR-145 in OA by directly targeting TNFRSF11B. These results suggested that controlling the expression of miR-145 offers potential as a novel approach for the prevention and treatment of OA.

\section{Acknowledgements}

The study was supported by the Natural Science Foundation of Shandong Province, China (grant no. ZR2010HQ036).

\section{References}

1. Miyaki S and Asahara H: Macro view of microRNA function in osteoarthritis. Nat Rev Rheumatol 8: 543-552, 2012.

2. Wu C, Tian B, Qu X, Liu F, Tang T, Qin A, Zhu Z and Dai K: MicroRNAs play a role in chondrogenesis and osteoarthritis (review). Int J Mol Med 34: 13-23, 2014.

3. Ambros V: The functions of animal microRNAs. Nature 431: 350-355, 2004.

4. Kim VN: Small RNAs: Classification, biogenesis, and function. Mol Cells 19: 1-15, 2005.

5. Bartel DP: MicroRNAs: Target recognition and regulatory functions. Cell 136: 215-233, 2009.

6. Dong S, Yang B, Guo H and Kang F: MicroRNAs regulate osteogenesis and chondrogenesis. Biochem Biophys Res Commun 418: 587-591, 2012.

7. Dunn W, DuRaine G and Reddi AH: Profiling microRNA expression in bovine articular cartilage and implications for mechanotransduction. Arthritis Rheum 60: 2333-2339, 2009.
8. Iliopoulos D, Malizos KN, Oikonomou P and Tsezou A: Integrative microRNA and proteomic approaches identify novel osteoarthritis genes and their collaborative metabolic and inflammatory networks. PLoS One 3: e3740, 2008.

9. Larne O, Hagman Z, Lilja H, Bjartell A, Edsjö A and Ceder Y: miR-145 suppress the androgen receptor in prostate cancer cells and correlates to prostate cancer prognosis. Carcinogenesis 36: 858-866, 2015

10. Bai M, Yuan M, Liao H, Chen J, Xie B, Yan D, Xi X, Xu X, Zhang Z and Feng Y: OCT4 pseudogene 5 upregulates OCT4 expression to promote proliferation by competing with miR-145 in endometrial carcinoma. Oncol Rep 33: 1745-1752, 2015.

11. Kim TH, Song JY, Park H, Jeong JY, Kwon AY, Heo JH, Kang H, Kim G and An HJ: miR-145, targeting high-mobility group A2, is a powerful predictor of patient outcome in ovarian carcinoma. Cancer Lett 356: 937-945, 2015.

12. Schmittgen TD and Livak KJ: Analyzing real-time PCR data by the comparative C(T) method. Nat Protoc 3: 1101-1108, 2008.

13. Zhang Y, Jia J, Yang S, Liu X, Ye S and Tian H: MicroRNA-21 controls the development of osteoarthritis by targeting GDF-5 in chondrocytes. Exp Mol Med 46: e79, 2014.

14. Iannone F and Lapadula G: The pathophysiology of osteoarthritis. Aging Clin Exp Res 15: 364-372, 2003.

15. Mortellaro CM: Pathophysiology of osteoarthritis. Vet Res Commun 27 (Suppl 1): S75-S78, 2003.

16. Martel-Pelletier J: Pathophysiology of osteoarthritis. Osteoarthritis Cartilage 12 (Suppl A): S31-S33, 2004.

17. Mandelbaum B and Waddell D: Etiology and pathophysiology of osteoarthritis. Orthopedics 28 (2 Suppl): S207-S214, 2005.

18. Golovchenko S, Hattori T, Hartmann C, Gebhardt M, Gebhard S, Hess A, Pausch F, Schlund B and von der Mark K: Deletion of beta catenin in hypertrophic growth plate chondrocytes impairs trabecular bone formation. Bone 55: 102-112, 2013

19. Feng ZY, He ZN, Zhang B and Chen Z: Osteoprotegerin promotes the proliferation of chondrocytes and affects the expression of ADAMTS-5 and TIMP-4 through MEK/ERK signaling. Mol Med Rep 8: 1669-1679, 2013.

20. Liang QQ, Li XF, Zhou Q, Xing L, Cheng SD, Ding DF, Xu LQ, Tang DZ, Bian Q, Xi ZJ, et al: The expression of osteoprotegerin is required for maintaining the intervertebral disc endplate of aged mice. Bone 48: 1362-1369, 2011. 